Case Report

\title{
Topical and Intranasal Analgesic Therapy in a Woman with Refractory Postherpetic Neuralgia
}

\author{
Kenneth C. Hohmeier ${ }^{1}$ and Lyndsey M. Almon ${ }^{2}$ \\ ${ }^{1}$ Department of Clinical Pharmacy, University of Tennessee Health Science Campus, 193 Polk Avenue, Suite 2D, \\ Nashville, TN 37210, USA \\ ${ }^{2}$ University of Tennessee Health Science Campus, 193 Polk Avenue, Suite 2D, Nashville, TN 37210, USA
}

Correspondence should be addressed to Kenneth C. Hohmeier; khohmeie@uthsc.edu

Received 5 March 2015; Accepted 25 March 2015

Academic Editor: Albert Dahan

Copyright (c) 2015 K. C. Hohmeier and L. M. Almon. This is an open access article distributed under the Creative Commons Attribution License, which permits unrestricted use, distribution, and reproduction in any medium, provided the original work is properly cited.

\begin{abstract}
A patient-specific, stepped approach to topical and intranasal analgesic pharmacotherapy was effective in reducing refractory postherpetic neuralgia (PHN) not responding to the current standard of care for PHN. The use of topical analgesic therapy allowed for higher concentrations of medication locally while reducing the likelihood of systemic side effects common to the drugs used. No adverse effects were noted for either topical or intranasal drug therapy. The patient-specific, stepped approach resulted in clinically significant decreases in pain on visual analog scale (VAS), with the use of intranasal ketamine $10 \%$ solution and topical gabapentin $6 \%$, ketoprofen $10 \%$, lidocaine $5 \%$, and ketamine $10 \%$ cream.
\end{abstract}

\section{Introduction}

New estimates report nearly 16 million people in the United States suffer from neuropathic pain $[1,2]$, defined as pain due to a damaged peripheral or central nerve [3] caused by a primary lesion or dysfunction in the nervous system [4]. Of these 3.8 million individuals, as many as 1 million suffer from postherpetic neuralgia (PHN) [5], a chronic complication of herpes zoster infection. Commonly referred to as shingles, herpes zoster results from the reactivation of the varicellazoster virus usually contracted during childhood in the form of the chickenpox $[6,7]$. The virus that had remained dormant in a sensory neuron remanifests as a painful, blistering, and vesicular rash along a dermatome [8] or an area of skin innervated by a single spinal nerve [6].

The reported prevalence of herpes zoster ranges between 1.5 and 4 cases per 1000 persons annually [9]. However, this frequency increases at the age of 50 years and older $[8,10]$, and over $50 \%$ of herpes zoster cases occur in persons $\geq 60$ years [9]. By age 90, the incidence rate is as high as 11 cases per 1000 people $[7,10]$, with a lifetime risk of developing the disease approximated at $30 \%$ [6]. The recurrence rate of an additional herpes zoster infection has not been clearly established, yet it is generally perceived to be similar to the rates of a first herpes zoster occurrence. Tseng et al. estimated the incidence rate to be significantly lower than first-occurrence rates at 7.48 cases per 1000 person-years in immunocompetent patients [11], while Yawn et al. report an estimated overall 8-year recurrence rate as high as $6.2 \%$ in a mixed immunocompetent and immunocompromised study group [12].

While the hallmark lesioned rash of herpes zoster usually clears within 2 to 4 weeks, $20 \%$ of people may experience pain in the form of PHN that persists after the rash has healed $[6,7]$. The pain is a consequence of peripheral nerve damage caused by the herpes zoster attack [13]. The duration of pain required for a diagnosis of PHN varies greatly from 1 to 9 months after the onset of the rash $[6,14]$ and can persist for years. In fact, $15 \%$ of patient report pain at 2 years [7]. Reported risk factors for PHN include advanced age, female gender, chronic disease, immunocompromised condition, and a greater severity of outbreak and pain during the acute phase $[6,7]$.

The pain experienced in PHN is often refractory to therapy, with as many as half of patients failing to respond to 
any treatment; other patients may experience limited efficacy despite being on multiple agents [6]. Treatment options to date have mainly been centered on oral therapies, including tricyclic antidepressants (TCAs), opioid analgesics, corticosteroids, and anticonvulsants (gabapentin, pregabalin). Therapeutic doses of these oral medications often carry with them a high risk of adverse effects and even addiction. Other current treatment options for PHN already include topical regimens, like lidocaine and capsaicin [7].

According to White et al., herpes zoster patients with PHN have a mean of 17.1 prescriptions filled compared to 5.5 prescriptions for patients without PHN [15]. With limited effective treatments, complicated dosing titration schedules, and overall low patient satisfaction, a gap in care is evident for this patient population [16]. It has been suggested in the literature that a topical, multimodal stepped-care approach to treating refractory pain should be considered [17]. This paper is the first case report discussing the use of such a stepped, patient-specific approach to treatment using a multimodal topical compounded analgesic cream in combination with intranasal ketamine to successfully reduce pain associated with refractory PHN.

\section{Case Report}

On July 26, 2012, a 78-year-old African American woman was referred to our pharmacotherapy consult service with a diagnosis of refractory postherpeticneuralgia $(\mathrm{PHN})$ of the head and neck. This was a recurrent episode of PHN following an original diagnosis in 2010. Pain was noted along the right sternocleidomastoid and masseter muscles per the referring physician. Insomnia and difficulty in speech secondary to pain were also noted. Other relevant medical conditions were rheumatoid arthritis and bilateral osteoarthritis of the knees. Past surgical history included bilateral knee replacements.

Medication history included previous treatment with oral amitriptyline and topical capsaicin $0.025 \%$, neither of which was tolerated by the patient and had been consequently discontinued. Oral amitriptyline was discontinued due to cognitive dysfunction and fatigue. Topical capsaicin was not tolerated due to local pain and discomfort on application of the cream. Her current medication regimen consisted of oxycodone, $20 \mathrm{mg}$ by mouth twice daily, gabapentin, $300 \mathrm{mg}$ by mouth three times daily, oxcarbazepine, $150 \mathrm{mg}$ by mouth three times daily, and tocilizumab administered intravenously using weight-based dosing once monthly. Further increases in titration to the oral gabapentin and oxcarbazepine were not possible due to patient-reported adverse effects.

When the patient presented to our pharmacotherapy consult service, she reported intermittent neuropathic pain that "comes and goes" over the past 2-3 years. According to the patient, this most recent exacerbation had been the worst in her memory. The patient described the pain as "electric," "tingling," and occurring with "bursts of lightning," which originated and were concentrated at the base of the jaw and which subsequently radiated up the lateral and posterior right sides of the cranium. She complained of skin and oral mucosal sensitivity, which was painful to the touch, on the inside and outside of her right cheek. The patient further complained of pain on swallowing, which had lead to the inability to eat full meals. She also complained of insomnia due to persistent baseline pain throughout the night. Severe pain symptoms were described as intermittent, lasting from minutes to hours, with several reported episodes of intense pain surrounding baseline pain. Despite the patient's own description of two separate types of paina baseline pain and an intermittent severe pain-on a VAS, she reported both types of pain as "10" on a 10-point visual analog scale (VAS). Due to the acutely severe and intense pain and the patient's severe decline in quality of life, it was decided that the addition of a lidocaine 5\% patch alone would likely be insufficient for pain relief, although in most situations a trial of topical lidocaine $5 \%$ would have been the recommended next step in treatment. The treating team decided to continue her baseline oral medications and also to initiate immediate treatment with a compounded combination topical analgesic cream composed of gabapentin $6 \%$, ketoprofen $10 \%$, amitriptyline $2 \%$, and lidocaine $5 \%$. The team's anecdotal successful experiences with this combination on other neuropathic pain syndromes led to this initial step. Patient directions were to apply sparingly to the face and neck at the site of pain no more than four times daily, avoiding the area around the eyes. Follow-up was to be conducted by the pharmacy team three days following the initiation of treatment and conveyed to the prescribing physician. Due to the time needed to compound the topical cream, the patient did not begin treatment until the $27 \mathrm{th}$ of July.

At the first patient follow-up on the 30th of July, the patient reported a decrease in baseline pain to $7 / 10$ on a VAS; however, peak pain remained constant at 10/10. The patient described her baseline pain as "quieted down" on cream but complained that pain "flare-ups" still remained a problem. At this point, the referring physician and consulting pharmacist made a change to a new topical analgesic cream formulation of gabapentin $6 \%$, ketoprofen $10 \%$, lidocaine $5 \%$, and ketamine $10 \%$. Topical ketamine replaced the amitriptyline due to the patient's concern of side effects based on her previous experience with the amitriptyline oral preparation. An oral mucosal topical analgesic gel was added as well. The antineuropathic agent used was again gabapentin in $10 \%$ concentration compounded with commercially available Orabase (a gel with benzocaine 20\%). Follow-up with the consulting pharmacist was scheduled for one week after the initiation of the new treatment.

At the next follow-up, the patient reported further decreases to the overall pain. The patient described pain as being at a tolerable level, which allowed her to sleep through the night and 5/10 on a VAS. However, breakthrough pain was still reported to be $8 / 10$. The previous topical regimens were continued, and the addition of a ketamine $100 \mathrm{mg} / \mathrm{mL}(10 \%)$ metered-dose intranasal spray delivering $0.1 \mathrm{~mL} /$ spray was initiated. Patient directions were to inhale 1 spray $(0.1 \mathrm{~mL})$, alternating nostrils 90 seconds apart, up to three times daily (with a maximum of 5 sprays per dose) for breakthrough pain. The patient was asked to lie in a supine position with her neck extended at a 45 -degree angle and to maintain this position for 30 seconds after 
administration. This dosing recommendation was based on previously successful intranasal ketamine therapy already reported in the literature [18].

Two weeks later, the patient reported further reduction in pain both at rest and when speaking, eating, and drinking. Breakthrough pain was managed on the intranasal ketamine regimen, and an overall reduction in baseline pain to $4 / 10$ was experienced with combination, multimodal pain treatment. The patient reported breakthrough pain relief on intranasal ketamine within 2-5 minutes after administration, on average 2-4 sprays (0.2-0.4 mLs) of the solution. Throughout the duration of therapy, no adverse effects were reported for either the nasal or topical therapies. Therapy was continued for the next several months until full, spontaneous remission of pain.

On November 7, 2013, the patient requested a refill of all medications secondary to a "flare-up." Due to the patient's age and concomitant immunosuppressive therapy with tocilizumab, recurrent PHN was confirmed on examination. This was the patient's third reported recurrence of PHN. The patient's previous three-prescription regimen was ordered. Again the patient reported a response to treatment, reporting pain score reduction on the VAS from 10/10 to 5/10.

\section{Discussion}

Treatment for postherpetic neuralgia (PHN) is based exclusively on symptom control and targeting the mechanisms causing pain. Oral treatment for PHN can typically include tricyclic antidepressants (TCAs), opioids analgesics, and anticonvulsants. TCAs block sodium, calcium, and NMDA receptors and inhibit serotonin and norepinephrine reuptake [19]. Opioids are mu-receptor agonists and have been shown to alleviate certain neuropathies $[13,20]$. Raja et al. investigated these two oral agents by comparing TCAs (nortriptyline $89 \mathrm{mg}$ or desipramine $63 \mathrm{mg}$ ) and opioids (morphine $91 \mathrm{mg}$ or methadone $15 \mathrm{mg}$ ) and placebo. In his screening of 103 patients, he found that TCAs decreased pain by $32 \%$, while opioids faired mildly better with a $38 \%$ improvement. Side effects of both TCAs and opioids are most often cited as reasons for cessation of treatment [21]. Gabapentin and pregabalin treat neuropathies by binding and blocking subunits of calcium channels in neurons, although systemic side effects often limit their use [22]. A recent systematic review of oral gabapentin showed that doses between 1800 and $3600 \mathrm{mg}$ daily provided significant improvement of PHN versus placebo [23]. However, Johnson et al. [24] conducted a retrospective claims database study of 1645 patients to examine real-world treatment with gabapentin $(N=939)$ and pregabalin $(N=706)$ for PHN. He found that over $50 \%$ of the patients switched from their original therapies in the treatment observation period of 12 months. Over one-third of those $50 \%$ of patients (35\% gabapentin, $31 \%$ pregabalin) switched to an opioid-based regimen. Also, $37 \%$ of the gabapentin index group and $31 \%$ of the pregabalin index group added medications to their regimens [24]. Oxcarbazepine monotherapy has also been investigated via an 8-week trial in 24 patients at a maintenance dose of $900 \mathrm{mg}$ orally per day and was shown to significantly reduce the mean VAS score, although mild to moderate side effects were noted [25].

Two commercially available topical agents have been investigated for the treatment of PHN. Both lidocaine and capsaicin are attractive options because their topical route of administration produces relatively low side-effect profiles and low systemic absorption [7]. Topical capsaicin affects the TRPV1 receptor, eventually leading to depletion of substance $\mathrm{P}$, a neurotransmitter known to cause pain and inflammation, from sensory neurons $[14,26]$. Topical lidocaine blocks voltage-gated sodium channels [14]. However, like the oral agents, efficacy of topical agents has also been unimpressive. Meier et al. did find a statistically significant decrease in pain with a $5 \%$ lidocaine patch, but any conclusions may be questionable as the placebo group reached a significant decrease in pain as well [27]. Capsaicin $0.075 \%$ decreased pain marginally by a maximum of $23-30 \%$ [28, 29]. The burning adverse reaction on application may limit this treatment, although pretreatment application of lidocaine cream may diminish this side effect.

Our hypothesis was that treatment with a stepped approach using patient-specific, multimodal topical therapy aimed at these pharmacological targets would achieve a reduction in reported pain on a VAS without the adverse effects common in oral therapies. This case report illustrates the use of a stepped approach to refractory PHN using a multimodal topical cream and single-ingredient ketamine intranasal solution. A review of the published evidence for topical therapy in PHN guided our stepped approach. The use of topical NSAIDS and depolarizing anesthetics, such as lidocaine and benzocaine, has been previously investigated in postherpetic nerve pain [30,31]. Topical gabapentin has been shown to be effective for PHN at concentrations of 6\% [32].

Amitriptyline used topically has some limited evidence of effectiveness in the treatment of localized pain $[19,33]$. Although we were unable to use amitriptyline and ketamine in combination with this patient, several studies have demonstrated effectiveness with this combination when used topically for a variety of pain conditions [34-36]. Additionally, the pool of evidence for topical ketamine, as both a single agent and in combination with other topical agents, for neuropathic pain continues to grow $[19,36]$. It should also be noted that, in this case study, the ketamine used in all formulations was the bulk powder of the racemic form of the active pharmaceutical ingredient (Letco Medical, Decatur, AL).

The decision to add intranasal ketamine was made based on two studies: one study reporting a metered-dose intranasal spray with lidocaine used for trigeminal neuralgia and the other reporting safety and efficacy of intranasal ketamine for the treatment of breakthrough pain [18, 37]. Based on this literature, it was theorized that intranasal delivery of antineuropathic therapy could be successfully delivered to the trigeminal nerve and that ketamine could be used safely and effectively for breakthrough pain; both criteria are necessary for treating our patient effectively. As the patient's symptoms were similar to those seen in patients with trigeminal neuralgia, it seemed a logical target for this patient's refractory breakthrough pain. Anatomically, analgesics administered 
intranasally should target the trigeminal nerve via the mucosa of the middle turbinate. Therefore, it is likely that the effectiveness seen in our patient may be due to local effects as well as systemic effects.

To date, there have been no reports in the literature regarding the use of intranasal ketamine in patients with PHN. Treatment of PHN using NMDA antagonists has been described previously in the literature via the intravenous route of administration [38]. However, its use in noncancer pain via the rapid-acting intranasal route remains controversial because of its potential risk for addiction [39]. Despite this concern, a double-blind, placebo-controlled crossover trial evaluated the use of intranasal ketamine or placebo in 20 patients with chronic cancer and noncancer pain. Patients in the trial reported significantly lower breakthrough pain $(P<0.0001)$ with intranasal ketamine [18]. Onset of action was 10 minutes on average, with a duration of 60 minutes. Adverse reactions included transient dysgeusia, nasal irritation, rhinorrhea, and increases in blood pressure. No reports of visual or auditory hallucinations were noted on patient questionnaires.

This is the first case report describing the use of a multimodal cream and intranasal ketamine for breakthrough pain in the treatment of PHN. This multimodal cream, intranasal ketamine, or the combination of both should be investigated in larger, randomized, and placebo-controlled trials on the treatment of PHN.

\section{Conflict of Interests}

The authors declare that there is no conflict of interests regarding the publication of this paper.

\section{References}

[1] G. J. Bennett, "Neuropathic pain: an overview," in Molecular Neurobiology of Pain: Progress in Pain Research and Management, D. Borsook, Ed., vol. 9, pp. 109-113, IASP Press, Seattle, Wash, USA, 1997.

[2] B. P. Yawn, P. C. Wollan, T. N. Weingarten, J. C. Watson, W. M. Hooten, and L. J. Melton III, "The prevalence of neuropathic pain: clinical evaluation compared with screening tools in a community population," Pain Medicine, vol. 10, no. 3, pp. 586593, 2009.

[3] K. E. Galluzzi, "Managing neuropathic pain," The Journal of the American Osteopathic Assoiation, vol. 107, no. 10, supplement 6, pp. 39-48, 2007.

[4] H. Merskey and N. Bogduk, Eds., Classification of Chronic Pain: Descriptions of Chronic Pain Syndromes and Definition of Pain Terms, IASP Press, Seattle, Wash, USA, 2nd edition, 1994.

[5] D. Bowsher, "The lifetime occurrence of Herpes zoster and prevalence of post-herpetic neuralgia: a retrospective survey in an elderly population," European Journal of Pain, vol. 3, no. 4, pp. 335-342, 1999.

[6] G. M. Sacks, "Unmet need in the treatment of postherpetic neuralgia.," The American Journal of Managed Care, vol. 19, supplement 1, pp. S207-S213, 2013.

[7] R. W. Johnson and A. S. Rice, "Postherpetic neuralgia," The New England Journal of Medicine, vol. 371, no. 16, pp. 1526-1533, 2014.
[8] R. W. Johnson, D. Bouhassira, G. Kassianos, A. Leplège, K. E. Schmader, and T. Weinke, "The impact of herpes zoster and post-herpetic neuralgia on quality-of-life," BMC Medicine, vol. 8, article 37, 2010.

[9] M. S. Bader, "Herpes zoster: diagnostic, therapeutic, and preventive approaches," Postgraduate Medicine, vol. 125, no. 5, pp. 78-91, 2013.

[10] R. P. Insinga, R. F. Itzler, J. M. Pellissier, P. Saddier, and A. A. Nikas, "The incidence of herpes zoster in a United States administrative database," Journal of General Internal Medicine, vol. 20, no. 8, pp. 748-753, 2005.

[11] H. F. Tseng, M. Chi, N. Smith, S. M. Marcy, L. S. Sy, and S. J. Jacobsen, "Herpes zoster vaccine and the incidence of recurrent herpes zoster in an immunocompetent elderly population," The Journal of Infectious Diseases, vol. 206, no. 2, pp. 190-196, 2012.

[12] B. P. Yawn, P. C. Wollan, M. J. Kurland, J. L. St Sauver, and P. Saddier, "Herpes zoster recurrences more frequent than previously reported," Mayo Clinic Proceedings, vol. 86, no. 2, pp. 88-93, 2011.

[13] S. H. Sindrup and T. S. Jensen, "Efficacy of pharmacological treatments of neuropathic pain: an update and effect related to mechanism of drug action," Pain, vol. 83 , no. 3, pp. 389-400, 1999.

[14] A. L. Cunningham and R. H. Dworkin, "The management of post-herpetic neuralgia," British Medical Journal, vol. 321, no. 7264, pp. 778-779, 2000.

[15] R. R. White, G. Lenhart, P. K. Singhal et al., "Incremental 1-year medical resource utilization and costs for patients with herpes zoster from a set of US health plans," PharmacoEconomics, vol. 27, no. 9, pp. 781-792, 2009.

[16] G. Oster, G. Harding, E. Dukes, J. Edelsberg, and P. D. Cleary, "Pain, medication use, and health-related quality of life in older persons with postherpetic neuralgia: results from a populationbased survey," The Journal of Pain, vol. 6, no. 6, pp. 356-363, 2005.

[17] D. J. Kopsky and J. M. Keppel Hesselink, "High doses of topical amitriptyline in neuropathic pain: two cases and literature review," Pain Practice, vol. 12, no. 2, pp. 148-153, 2012.

[18] D. B. Carr, L. C. Goudas, W. T. Denman et al., "Safety and efficacy of intranasal ketamine for the treatment of breakthrough pain in patients with chronic pain: a randomized, double-blind, placebo-controlled, crossover study," Pain, vol. 108, no. 1-2, pp. 17-27, 2004.

[19] N. B. Finnerup, M. Otto, H. J. McQuay, T. S. Jensen, and S. H. Sindrup, "Algorithm for neuropathic pain treatment: an evidence based proposal," Pain, vol. 118, no. 3, pp. 289-305, 2005.

[20] P. L. I. Dellemijn, H. Van Duijn, and J. A. L. Vanneste, "Prolonged treatment with transdermal fentanyl in neuropathic pain," Journal of Pain and Symptom Management, vol. 16, no. 4, pp. 220-229, 1998.

[21] S. N. Raja, J. A. Haythornthwaite, M. Pappagallo et al., "Opioids versus antidepressants in postherpetic neuralgia: a randomized, placebo-controlled trial," Neurology, vol. 59, no. 7, pp. 1015-1021, 2002.

[22] J. D. Gale and L. A. Houghton, "Alpha 2 delta $(\alpha 2 \delta)$ ligands, gabapentin and pregabalin: what is the evidence for potential use of these ligands in irritable bowel syndrome," Frontiers in Pharmacology, vol. 2, article 28, 2011.

[23] R. A. Moore, P. J. Wiffen, S. Derry, and H. J. McQuay, "Gabapentin for chronic neuropathic pain and fibromyalgia in 
adults," Cochrane Database of Systematic Reviews, no. 3, Article ID CD007938, 2011.

[24] P. Johnson, L. Becker, R. Halpern, and M. Sweeney, "Realworld treatment of post-herpetic neuralgia with gabapentin or pregabalin," Clinical Drug Investigation, vol. 33, no. 1, pp. 35-44, 2013.

[25] S. Criscuolo, C. Auletta, S. Lippi, F. Brogi, and A. Brogi, "Oxcarbazepine monotherapy in postherpetic neuralgia unresponsive to carbamazepine and gabapentin," Acta Neurologica Scandinavica, vol. 111, no. 4, pp. 229-232, 2005.

[26] H. Knotkova, M. Pappagallo, and A. Szallasi, "Capsaicin (TRPV1 agonist) therapy for pain relief: farewell or revival?” The Clinical Journal of Pain, vol. 24, no. 2, pp. 142-154, 2008.

[27] T. Meier, G. Wasner, M. Faust et al., "Efficacy of lidocaine patch $5 \%$ in the treatment of focal peripheral neuropathic pain syndromes: a randomized, double-blind, placebo-controlled study," Pain, vol. 106, no. 1-2, pp. 151-158, 2003.

[28] C. P. N. Watson, K. L. Tyler, D. R. Bickers, L. E. Millikan, S. Smith, and E. Coleman, "A randomized vehicle-controlled trial of topical capsaicin in the treatment of postherpetic neuralgia," Clinical Therapeutics, vol. 15, no. 3, pp. 510-526, 1993.

[29] J. E. Bernstein, N. J. Korman, D. R. Bickers, M. V. Dahl, and E. Millikan, "Topical capsaicin treatment of chronic postherpetic neuralgia," Journal of the American Academy of Dermatology, vol. 21, no. 2, pp. 265-270, 1989.

[30] G. de Benedittis, F. Besana, and A. Lorenzetti, "A new topical treatment for acute herpetic neuralgia and post-herpetic neuralgia: the aspirin/diethyl ether mixture. An open-label study plus a double-blind controlled clinical trial," Pain, vol. 48, no. 3, pp. 383-390, 1992.

[31] M. Padilla, G. T. Clark, and R. L. Merrill, "Topical medications for orofacial neuropathic pain: a review," The Journal of the American Dental Association, vol. 131, no. 2, pp. 184-195, 2000.

[32] S. Hiom, G. Patel, R. Newcombe, and S. Khot, "Severe postherpetic neuralgia and other neuropathic pain syndromes alleviated by topical gabapentin," British Journal of Dermatology, 2014.

[33] R. Liebregts, D. J. Kopsky, and J. M. Hesselink, "Topical amitriptyline in post-traumatic neuropathic pain," Journal of Pain and Symptom Management, vol. 41, no. 4, pp. e6-e7, 2011.

[34] J. S. Gewandter, S. G. Mohile, C. E. Heckler et al., "A phase III randomized, placebo-controlled study of topical amitriptyline and ketamine for chemotherapy-induced peripheral neuropathy (CIPN): a University of Rochester CCOP study of 462 cancer survivors," Supportive Care in Cancer, vol. 22, no. 7, pp. 1807-1814, 2014.

[35] T. J. Poterucha, S. L. Murphy, M. D. P. Davis et al., "Topical amitriptyline combined with ketamine for the treatment of erythromelalgia: a retrospective study of 36 patients at mayo clinic," Journal of Drugs in Dermatology, vol. 12, no. 3, pp. 308310, 2013.

[36] J. Sawynok, "Topical and peripheral ketamine as an analgesic," Anesthesia \& Analgesia, vol. 119, no. 1, pp. 170-178, 2014.

[37] A. Kanai, A. Suzuki, M. Kobayashi, and S. Hoka, "Intranasal lidocaine $8 \%$ spray for second-division trigeminal neuralgia," British Journal of Anaesthesia, vol. 97, no. 4, pp. 559-563, 2006.

[38] P. K. Eide, E. Jørum, A. Stubhaug, J. Bremnes, and H. Breivik, "Relief of post-herpetic neuralgia with the N-methylD-aspartic acid receptor antagonist ketamine: a double-blind, cross-over comparison with morphine and placebo," Pain, vol. 58, no. 3, pp. 347-354, 1994.
[39] R. F. Bell and E. Kalso, "Is intranasal ketamine an appropriate treatment for chronic non-cancer breakthrough pain?" Pain, vol. 108, no. 1, pp. 1-2, 2004. 


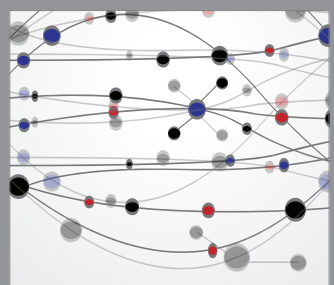

The Scientific World Journal
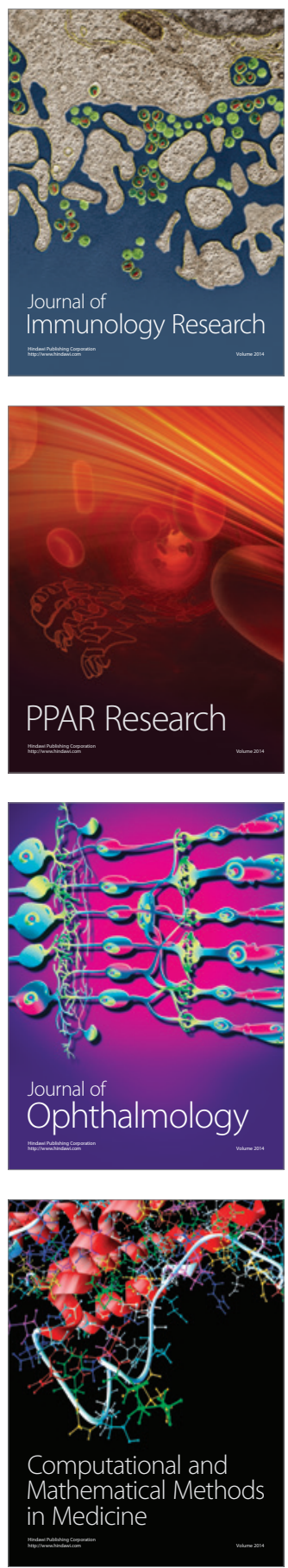

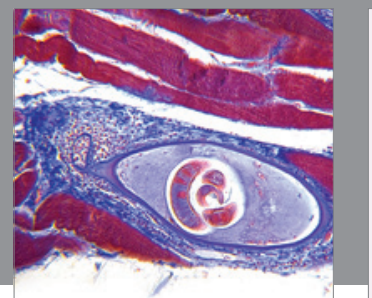

Gastroenterology

Research and Practice
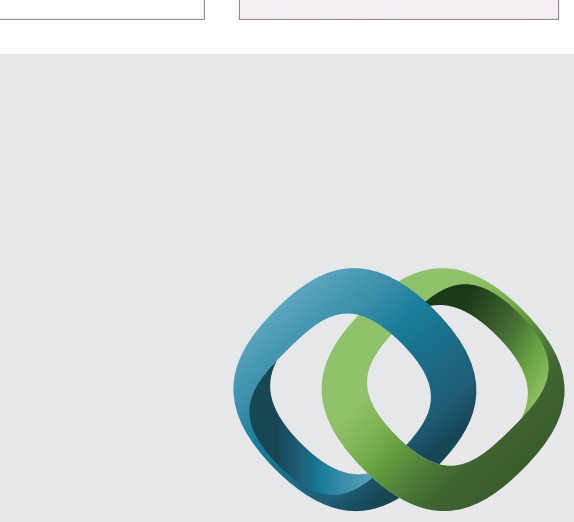

\section{Hindawi}

Submit your manuscripts at

http://www.hindawi.com
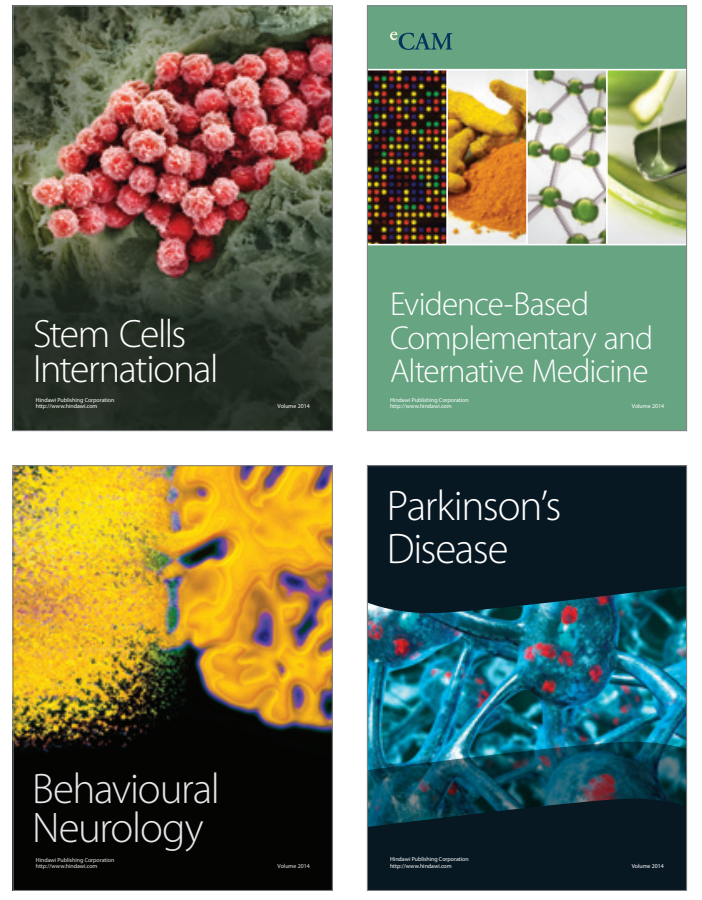
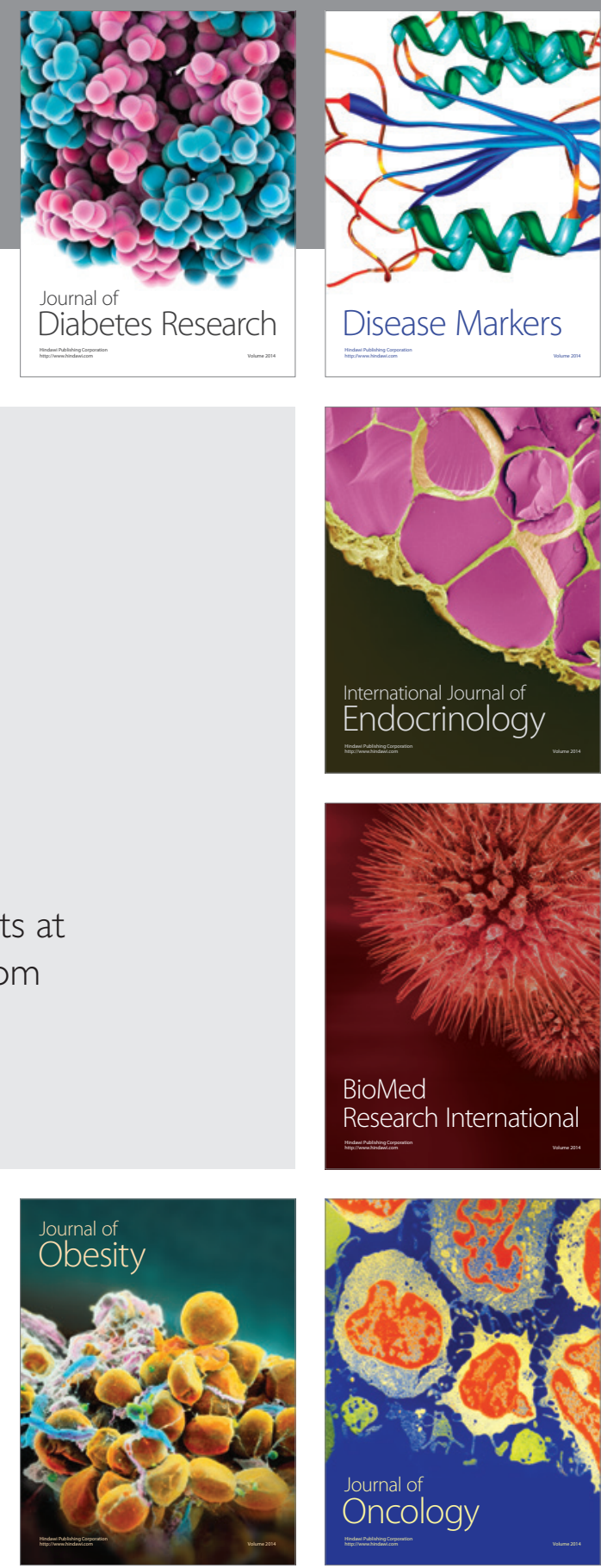

Disease Markers
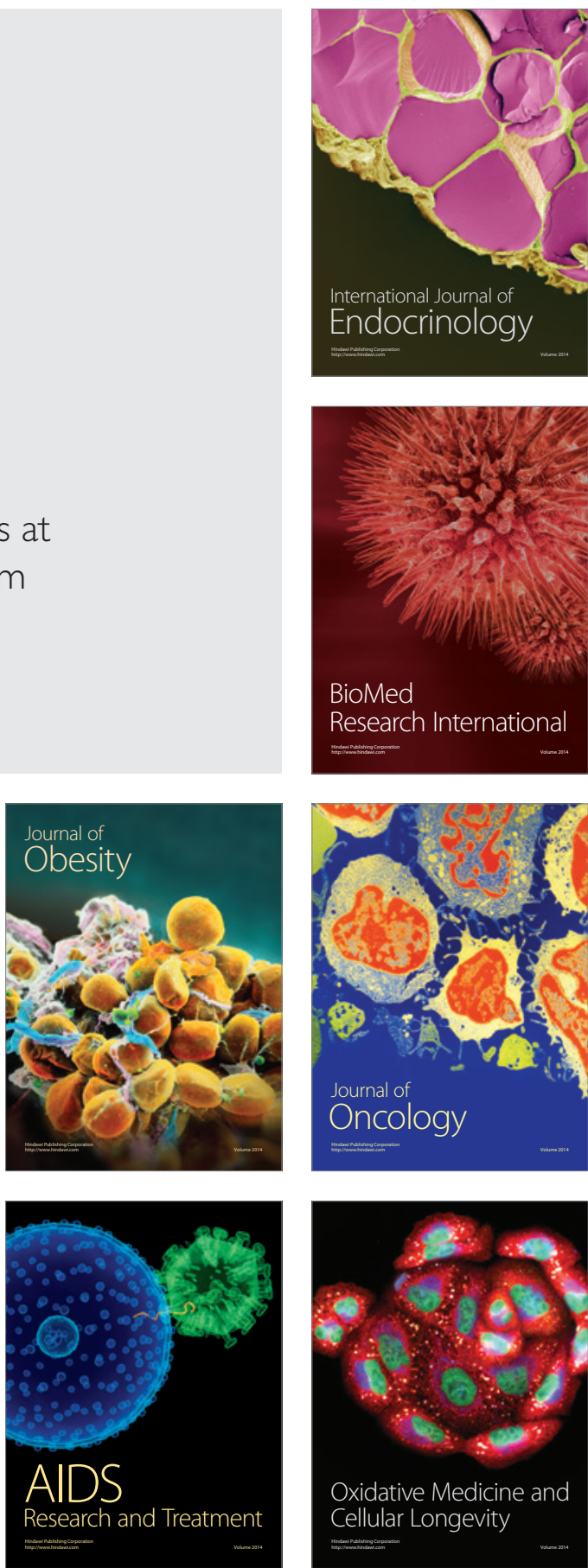